\title{
Photodynamic therapy associated with full-mouth ultrasonic debridement in the treatment of severe chronic periodontitis: a randomized-controlled clinical trial
} Maybel Lages BALATA 1 , Lyla Prates de ANDRADE ${ }^{2}$, David Barros Nunes SANTOS ${ }^{3}$, Andrea Nóbrega CAVALCANTI ${ }^{4}$,
Urbino da Rocha TUNES ${ }^{4}$, Érica Del Peloso RIBEIRO ${ }^{4}$, Sandro BITTENCOURT ${ }^{4}$

\footnotetext{
1- MS, Specialist in Periodontics, Bahiana School of Medicine and Public Health, Salvador, BA, Brazil

2- Specialist in Periodontics, Bahiana School of Medicine and Public Health, Salvador, BA, Brazil

3- Graduate student, Bahiana School of Medicine and Public Health, Salvador, BA, Brazil

4- DDS, MSc, PhD, Bahiana School of Medicine and Public Health, Salvador, BA, Brazil
}

Corresponding address: Sandro Bittencourt - Avenida Silveira Martins, n. 3386 - Cabula - 41150-100 - Salvador - BA - Brazil - Phone: 71-9156-1998 - Fax number: 71-3257-8230 - e-mail: sandrobittencourt@yahoo.com

Received: May 7, 2012 - Modification: January 4, 2013 - Accepted: January 31, 2013

\section{ABSTRACT}

$B^{\text {a }}$ ackground: Photodynamic therapy (PDT) is a method of microbial reduction which can benefit periodontal treatment in areas of difficult access, such as deep pockets and furcations. The aim of this randomized controlled clinical trial was to evaluate the effects of PDT as an adjunct to full-mouth ultrasonic debridement in the treatment of severe chronic periodontitis. Material and Methods: Twenty-two patients with at least one pocket with a probing depth (PD) of $\geq 7 \mathrm{~mm}$ and one pocket with a PD of $\geq 5 \mathrm{~mm}$ and bleeding on probing (BOP) on each side of the mouth were included, characterizing a split mouth design. The control group underwent full-mouth ultrasonic debridement and the test group received the same treatment associated with PDT. The PDT was performed on only one side of the mouth and the initial step consisted of subgingival irrigation with $0.005 \%$ methylene blue dye. Two minutes after applying the photosensitizer, the low power laser - AsGaAl (Photon Lase III - PL7336, DMC, São Carlos -São Paulo, Brazil) was applied (660 nm, 100 mW, 9 J, 90 seconds per site, $320 \mathrm{~J} / \mathrm{cm}^{2}$, diameter tip $\left.600 \mu \mathrm{m}\right)$. The following clinical parameters were evaluated: plaque index, gingival index, BOP, gingival recession (GR), PD, and clinical attachment level (CAL). All parameters were collected before, 1, 3 and 6 months after treatment. Results: An improvement in BOP, PD and CAL was observed after treatment, in both groups, but without any difference between them. After 6 months, the PD decreased from $5.11 \pm 0.56 \mathrm{~mm}$ to $2.83 \pm 0.47 \mathrm{~mm}$ in the test group $(p<0.05)$ and from $5.15 \pm 0.46$ $\mathrm{mm}$ to $2.83 \pm 0.40 \mathrm{~mm}$ in the control group $(\mathrm{p}<0.05)$. The CAL changed, after 6 months, from $5.49 \pm 0.76 \mathrm{~mm}$ to $3.41 \pm 0.84 \mathrm{~mm}$ in the test group $(p<0.05)$ and from $5.53 \pm 0.54$ to $3.39 \pm 0.51 \mathrm{~mm}$ in the control group $(\mathrm{p}<0.05)$. Conclusion: Both approaches resulted in significant clinical improvements in the treatment of severe chronic periodontits, however, the PDT did not provide any additional benefit to those obtained with full-mouth ultrasonic debridement used alone.

Key words: Laser. Clinical trial. Non-surgical periodontal therapy. Periodontitis.

\section{INTRODUCTION}

Periodontitis is an inflammatory reaction of the supporting tissues of the teeth that usually results from the extent of subgingival inflammation induced by bacteria in the biofilm ${ }^{4}$. Non-surgical treatment of periodontitis is traditionally performed with scaling and root planning (SRP) using manual instruments to remove supra and subgingival bacterial deposits.

In the last years, the full-mouth ultrasonic debridement raised as a new non-surgical treatment based on the knowledge that removing the tooth 
structure is not a prerequisite for periodontal healing, because bacterial lipopolysaccharide is easily removed from the root surface ${ }^{12,20}$. Nowadays, the periodontal debridement, defined by Smart, et al. ${ }^{24}$ (1990) as a conservative instrumentation regimen of overlapping strokes and light pressure used for only a limited time period, is considered an alternative for the treatment of chronic periodontitis ${ }^{10,29}$. The purpose of this approach is to produce a biologically compatible root surface for healthy attachment.

However, mechanical instrumentation has limitations and even with therapy, some patients still have attachment loss probably due to the persistence of periodontal pathogens and subsequent recolonization ${ }^{10}$. Thus, the advent of other options to improve the effectiveness of periodontal therapy is needed due to limited access to furcation areas, concavities, grooves, distal sites of molars and deep pockets found during conventional periodontal therapy ${ }^{5,26}$. The increase in bacterial resistance due to the use systemic antibiotics could also justify the appearance of other adjuvants for established periodontal treatment ${ }^{13,21}$.

In this context, photodynamic therapy (PDT) appears as a method for microbial reduction, with minimal side effects ${ }^{31}$. Thus, the existence of favorable effects of this therapy as an adjuvant method to conventional periodontal treatments was proposed. The procedure could be beneficial in areas of difficult access, reducing the need for flaps, treatment time and the risk of bacteremia ${ }^{31}$.

Photodynamic therapy associates low power laser with a photosensitizer that links to the target cells (periodontal pathogens). When activated by light with an appropriate wavelength the photosensitizer reacts with oxygen to produce an electronically excited and highly reactive state of oxygen, known as singlet oxygen, which can interact with a large number of biological substrates as a result of its high chemical reactivity. This interaction induces oxidative damage and ultimately lethal effects upon the bacterial cell. Singlet oxygen has a short lifetime in biological systems and a very short radius of action. These radicals are toxic to bacterial cells, destroying the membrane, mitochondria or the nucleus ${ }^{13,18,23,25,26}$.

In vitro studies showed complete elimination of anaerobic bacteria such as Porphyromonas gingivalis $(\mathrm{Pg})$, Fusobacterium nucleatum (Fn) and Capnocytophaga gingivalis $(\mathrm{Cg})$ after PDT ${ }^{22}$. Furthermore, Komerik, et al. ${ }^{15}$ (2000) and Zanim and Brugnera $\mathrm{Jr}^{31}$ (2007)reported a reduction in the activity of virulence factors such as lipopolysaccharides and proteases after PDT. Animal studies have also shown the suppressive effect on periodontal pathogens ${ }^{26}$ and the statistically significant decrease of bone loss after application of PDT2,3,16.

To verify the effect of PDT as an adjuvant to SRP, some clinical studies were conducted in patients with chronic periodontitis. Some authors such as Andersen, et al. ${ }^{4}$ (2007) and Braun, et al.7 (2008) reported that PDT associated with SRP promotes a statistically significant improvement in the reduction of probing depth, bleeding on probing and gain of clinical attachment level. However, Chondros, et al. ${ }^{8}$ (2009), Christodoulides, et al. ${ }^{9}$ (2008), Polansky, et al. ${ }^{23}$ (2009) did not find any statistically significant benefit in PD and CAL changes, favoring the test group submitted to SRP with PDT.

Atieh $^{6}$ (2010), after a systematic review and meta-analysis concluded that the combined use of PDT with conventional SRP may provide additional improvements in $C A L, P D$ and other clinical measures in the treatment of chronic periodontitis. However, it is still questionable if this improvement is clinically meaningful due to the limited amount of data.

In view of the theme relevance and the existence of conflicting results in the literature, the aim of the present study was to evaluate the clinical effects of the adjunctive use of PDT in the full-mouth ultrasonic debridement of patients with severe chronic periodontitis.

\section{MATERIAL AND METHODS}

\section{Study design}

The present study was a randomized, blinded, controlled clinical trial, which used a split-mouth design. Prior to commencement, the study design was approved by the Ethics Committee of Bahiana School of Medicine and Public Health (12/2008). All patients were informed individually about the nature of the proposed treatment, and informed consent forms were signed.

\section{Study population}

Twenty-two subjects from those referred for treatment to the Bahiana School of Medicine and Public Health, Salvador, BA, Brazil, were recruited after a screening examination that included a full medical and dental history, intraoral examination, full-mouth periodontal probing, and radiographs. Subjects were enrolled from February 2008 to October 2009.

The power calculation was performed after the pilot study by the SAS 9.1 statistical program (SAS Institute, Cary, NC, USA). The analysis indicated that with 11 individuals the study would have > $80 \%$ power to detect a difference of $1 \mathrm{~mm}$ in CAL between the two groups. Subjects who were invited to participate met the following inclusion criteria: 1) diagnosis of severe chronic periodontitis by the presence of periodontal pockets with clinical 
attachment loss $(\mathrm{CAL}) \geq 5 \mathrm{~mm}$, bleeding on probing (BOP) and radiographic bone loss ${ }^{11}$; 2) minimum of 2 teeth with probing depth (PD) $\geq 7 \mathrm{~mm}$ and 2 other teeth with a PD $\geq 5 \mathrm{~mm}$, all with BOP and located on opposite sides of the mouth; and 3) $\geq 16$ teeth in both jaws (wisdom teeth excluded).

Exclusion criteria were as follows: periapical alterations on qualifying teeth, systemic diseases that require prophylaxis antibiotic coverage or that could influence progression and response to treatment, periodontal treatment in the preceding 6 months, consumption of antibiotic, antiinflammatory, anticonvulsant, immunosuppressant or calcium channel blocker within the past 3 months; pregnancy; orthodontic therapy, smoking and cardiac pace-makers users.

\section{Treatment}

Initially, the patients received information about the etiology of periodontal disease and instructions for maintaining adequate plaque control, including interdental cleaning with dental floss and interdental toothbrushes (Bitufo). Furthermore, in the initial sessions, patients had the biofilm retentive factors (caries, excesses of restorations, supragingival calculus) removed and condemned teeth extracted. Initial measurements were obtained at least 15 days after this initial phase.

At least two teeth (one with a PD $\geq 7 \mathrm{~mm}$ and another with a PS $\geq 5 \mathrm{~mm}$ ) were randomly assigned (by coin toss) to one of the treatments: with (test group) or without PDT (control group). The allocation concealment was secured by having a person responsible for the treatment (L.P.A) and another person responsible for the examinations (M.L.B). The randomization code was not broken until all data had been collected. Thus, the treatment group was not revealed to the clinical examiner or to the statistician. After random allocation, all patients received full-mouth ultrasonic debridement using an ultrasonic scaler (Profi III Bios, Dabi Atlante, Ribeirão Preto, São Paulo, Brazil) for 1 hour. Specific tips were used (Perio sub, Dabi Atlante, Ribeirão Preto, SP, Brazi). PDT was performed on only one side of the mouth and the initial step was subgingival irrigation with $0.005 \%$ methylene blue dye. To avoid contamination of the control sites with the dye, the methylene blue was applied only inside the periodontal pockets and a high-powered suction device controlled the flow. Two minutes after applying the photosensitizer, the low power laser - AsGaAl (Photon Lase III - PL7336, DMC, São Carlos -SP, Brazil) was applied in a 90 degree angle with the gingival surface and with no contact with the tissues (660 nm, $100 \mathrm{~mW}, 9 \mathrm{~J}, 90$ seconds per site, $320 \mathrm{~J} / \mathrm{cm}^{2}$, diameter tip $600 \mu \mathrm{m}$ ).

After treatment, the patients were included in a supportive periodontal therapy program, in which they received weekly supragingival plaque control during the first month. After the first 4 weeks, monthly reinforcement of oral hygiene instructions and prophylaxis with prophylactic paste and rubber cups were performed in all the patients. This maintenance program also included updates in the medical and dental history, intra and extra-oral exams and periodontal evaluations. Three months after treatment, the remaining pockets with PD $\geq 5 \mathrm{~mm}$ and BOP were reinstrumented with Gracey curettes (Hu-Friedy, Chicago, IL, USA).

\section{Clinical measurements}

The following parameters were assessed at baseline and at 1, 3 and 6 months after therapy by a masked and calibrated examiner. The presence of supragingival biofilm and marginal gingival bleeding was evaluated using the visible plaque index $(\mathrm{VPI})^{1}$ and gingival bleeding index (GBI) dichotomously at four sites on all teeth in the mouth ${ }^{19}$. BOP was also measured dichotomously at six sites per tooth. Gingival recession (GR), probing depth (PD) and clinical attachment level (CAL) were measured using a periodontal probe with markings at intervals of $1 \mathrm{~mm}$ (15 PCPUNC ${ }^{\circledR}$ Hu-Friedy, Chicago, IL, USA).

\section{Examiner calibration}

The researcher in charge of the clinical evaluations (M.L.B.) was calibrated for intraexaminer repeatability before the beginning of the study. Four patients with chronic periodontitis were examined at an interval of 48 hours. The intraclass correlation coefficients as a measure of intraexaminer reproducibility were 0.89 and 0.90 for the mean PD and RAL. The calibration of the laser was performed at the Institute of Physics, Federal University of Bahia.

\section{Data management and statistical analyses}

The clinical parameters obtained in the experimental groups were tabulated for the statistical analysis. First an exploratory analysis of the data was made to verify the homogeneity of variances and to determine if the experimental errors had a normal distribution (parameters of analysis of variance). According to this analysis, the variable data of BOP needed to be transformed into its square root, and the variable GR had to be analyzed as a non-parametric variable. The inferential statistical analysis of the variables PI, GI, PD, BOP and CAL were performed by the analysis of variance in a split plot design, and the plot was represented by the "treatment" (two levels) and the sub-plot represented by "time" (four levels). The data was obtained by analysis of variance for the variable $\mathrm{PD}$, considering the deep and moderate pockets, indicated that this clinical parameter needed to be converted into a base 10 
logarithm. The data were analyzed in the SAS 9.1 statistical program (SAS Institute, Cary, NC, USA) with a level of significance fixed at $5 \%$. For the variable GR, the Wilcoxon test was used to compare treatments and the Friedman test to compare times. This analysis was conducted in the BioEstat program, version 5.0, with a level of significance of $5 \%$. The proportions of sites with RAL gain $\geq 2$ $\mathrm{mm}$ and the number of sites retreated at 3 and 6 months were compared between groups using the $\mathrm{X}^{2}$ test. All evaluations used the subject as the unit of measurement. Individual sites were compared with regard to RAL gain $\geq 2 \mathrm{~mm}$, and the number of sites referred for treatment. RAL was considered as the primary outcome variable. All other parameters were considered secondary outcomes.

\section{RESULTS}

No patients were excluded from the study. Thus, the sample was composed of 14 women and 8 men, totaling 22 patients with a mean age of 43.18 years (31-62 years). Figure 1 illustrates the study flowchart. The number of sites showing a baseline

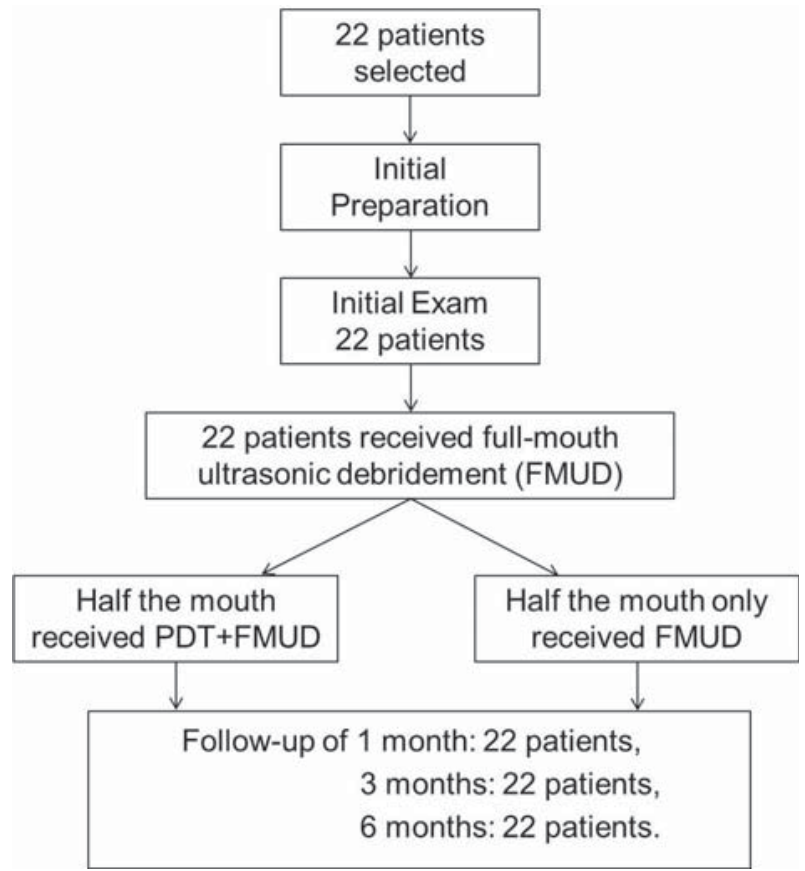

Figure 1- Flowchart of study design
PPD $\geq 5 \mathrm{~mm}$ and BOP (qualifying sites) were 40.18 $\%$ out of which $10.48 \%$ had a PPD $\geq 7$. The baseline data indicated that both groups were similar with regard to age, gender, and clinical parameters.

\section{VPI, GBI and BOP}

The oral hygiene status during the course of the study is illustrated in Table 1 . VPI showed a statistically significance $(p<0.05)$ reduction 1 and 6 months after treatment when compared with the initial values. There was no difference between 1, 3 and 6 months. GBI was significantly reduced after 3 and 6 months $(p<0.05)$. No differences were found between 1, 3 and 6 months. BOP was evaluated separately in the control and test groups (Table 2 ). This analysis showed that BOP was significantly reduced during the study without any difference between groups.

\section{GR, PD and CAL}

No difference was observed between groups regarding $G R, P D$ and $C A L$ at different time intervals. The increase in $\mathrm{GR}$ only reached a statistically significance $(p<0.05)$ in moderate pockets of the test group after 6 months (Table 3 ). Both groups showed a reduction in PD and a gain in CAL (Table 4). At 6 months, the PD decrease in the moderate and deep pockets was $1.96 \mathrm{~mm}$ and $3.84 \mathrm{~mm}$ for the test group and $2.03 \mathrm{~mm}$ and $4.24 \mathrm{~mm}$ for the control group, respectively. Also at this time interval the CAL gain in the moderate and deep pockets was $1.79 \mathrm{~mm}$ and $3.57 \mathrm{~mm}$ for the test group and $1.80 \mathrm{~mm}$ and $3.90 \mathrm{~mm}$ for the control group, respectively.

There was also no statistical difference between groups considering CAL gain $\geq 2 \mathrm{~mm}$. After 3 months, $62.47 \%$ of sites in the test group and $58.95 \%$ of sites in the control group showed CAL gain $\geq 2 \mathrm{~mm}$. These values increased to $68.88 \%$ and $68.12 \%$ after 6 months in the test and control groups, respectively. The percentage of sites that needed retreatment at 3 months was $7.83 \%$ in the test group and $8.95 \%$ in the control group $(p>0.05)$. At 6 months, these values were $4.51 \%$ and $4.16 \%(p>0.05)$, respectively. No adverse effect was reported by patients of any group.

Table 1- Mean (standard deviation) of the variables VPI and GBI considering the entire mouth, at different time intervals

\begin{tabular}{ccccc}
\hline & Baseline & 1 month & 3 months & $\mathbf{6}$ months \\
\hline VPI & $18.83(9.35)$ & $12.50(7.60)$ & $16.68(7.87)$ & $13.60(7.85)$ \\
& $\mathrm{a}$ & $\mathrm{b}$ & $\mathrm{ab}$ & $\mathrm{b}$ \\
$\mathrm{GBI}$ & $37.48(18.06)$ & $30.73(17.34)$ & $27.86(16.85)$ & $25.37(14.28)$ \\
& $\mathrm{a}$ & $\mathrm{ab}$ & $\mathrm{b}$ & $\mathrm{b}$ \\
\hline
\end{tabular}

Different letters represent a statistical significance (lowercase letters compare times; ANOVA in split plot design/Tukey) 
Table 2- Mean (standard deviation) of bleeding on probing (BOP) at different time intervals for both groups

\begin{tabular}{|c|c|c|c|c|c|c|}
\hline & & Baseline & 1 month & 3 months & 6 months & \\
\hline \multirow[t]{3}{*}{ BOP } & Test group & $61.58(15.64)$ & $46.16(21.18)$ & $40.67(20.63)$ & $36.73(19.72)$ & $A$ \\
\hline & Control group & $62.23(16.91)$ & $47.41(21.48)$ & $42.21(21.42)$ & $38.49(20.53)$ & A \\
\hline & & $a$ & $\mathrm{~b}$ & $\mathrm{bc}$ & $\mathrm{C}$ & \\
\hline
\end{tabular}

Different letters represent a statistical significance (lowercase letters compare times and uppercase letters compare treatments; ANOVA in split plot design/Tukey)

Table 3- Median values of the variable GR $(\mathrm{mm})$, in baseline moderate $(5-6 \mathrm{~mm})$ and deep pockets $(\geq 7 \mathrm{~mm})$ at different time intervals for control and test groups

\begin{tabular}{ccccccc}
\hline & & & Baseline & 1 month & 3 months & $\mathbf{6}$ months \\
\hline Moderate & GR & Test group & $1.5^{\mathrm{Ab}}$ & $2^{\mathrm{Aab}}$ & $1.8^{\mathrm{Aab}}$ & $2^{\mathrm{Aa}}$ \\
pockets & & Control group & $2^{\mathrm{Aa}}$ & $1.9^{\mathrm{Aa}}$ & $2^{\mathrm{Aa}}$ & $1.8^{\mathrm{Aa}}$ \\
Deep pockets & $\mathrm{GR}$ & Test group & $0^{\mathrm{Aa}}$ & $1^{\mathrm{Aa}}$ & $1.2^{\mathrm{Aa}}$ & $1^{\mathrm{Aa}}$ \\
& & Control group & $0^{\mathrm{Aa}}$ & $0.7^{\mathrm{Aa}}$ & $0.5^{\mathrm{Aa}}$ & $0.5^{\mathrm{Aa}}$ \\
\hline
\end{tabular}

Medians followed by different letters represent statistical significance (uppercase letters compare treatments and lowercase letters compare times); Wilcoxon/Friedman)

\section{DISCUSSION}

Access limitations during non-surgical periodontal treatment such as furcation areas, concavities, grooves, distal sites of molars and deep pockets led to the appearance of adjuvant options for periodontal therapy such as PDT ${ }^{5,26}$. Therefore, the aim of the present study was to evaluate the effect of this treatment in patients with severe chronic periodontitis. Despite the existent biological plausibility in PDT, the results of the present study showed that PDT does not provide additional clinical benefit to non-surgical periodontal therapy.

The results revealed improvements in BOP, $\mathrm{PD}$ and $\mathrm{CAL}$ after both treatments, but there was no difference between the groups in any of the clinical parameters assessed. These results are similar to those found by Yilmaz, et al. ${ }^{28}$ (2002) who observed intra-group differences, but affirmed that SRP + PDT did not cause major clinical and microbiological benefits to those achieved with SRP alone. However, Yilmaz, et al. ${ }^{28}$ (2002) used a reduced sample (10 patients), with low requirements in the inclusion criteria (1 uniradicular tooth in each quadrant with PD $\geq 4 \mathrm{~mm}$ ).

Similar results to the ones in the present study were also obtained by Christodoulides, et al. ${ }^{9}$ (2008) It was found that a single application of PDT associated with SRP failed to improve PD, $\mathrm{CAL}$ and the amount of periodontal pathogens. However, the authors observed a high reduction of full-mouth bleeding index in the test group when compared with the control group, 3 and 6 months after therapy. This was not confirmed in the present study probably due to the difference in study design, since Christodoulides, et al. ${ }^{9}$ (2008) used a parallel design and the present study had a split mouth design.

Also Chondros, et al. ${ }^{8}$ (2009), in evaluating the clinical and microbiological effectiveness of SRP+PDT in periodontal patients under maintenance, affirmed that PDT failed to improve further PD reduction and gain CAL. Again, a significant decrease in BOP in the test group was reported when compared with the control group. However, this information has to be seen with caution since smokers were included in the study. Similarly, Polansky, et al. ${ }^{23}$ (2009) concluded that a single application of PDT was incapable producing additional clinical and microbiological effects when associated with periodontal instrumentation.

The absence of additional benefits with PDT, in the present study, could be explained by the significant improvements observed in the control group, including the good plaque control maintained during the entire observation period of the study. The low PI values were due to the protocol of supragingival plaque control performed weekly during the first month after treatment and biweekly during the other 5 months. The commitment of patients to treatment was essential to assess the effect of PDT. Only the studies of Christodoulide, et al. ${ }^{9}(2008)$ and Chondros, et al. ${ }^{8}$ (2009) showed similar PI to the ones found in the present study.

Thus, the results obtained in the control group were within the range expected from this nonsurgical approach ${ }^{10,29,30}$. Six months after full-mouth ultrasonic debridement, PD reduction was 2.03 $\mathrm{mm}$ and $4.24 \mathrm{~mm}$ for the initially moderate and deep pockets, respectively. At the same time, CAL 
gain was $1.80 \mathrm{~mm}$ and $3.90 \mathrm{~mm}$, respectively. Six months after full-mouth ultrasonic debridement Del Peloso Ribeiro, et al. ${ }^{10}(2008)$ reported PD changes of 1.93 and $3.44 \mathrm{~mm}$ and CAL gain of 1.21 and 2.41 $\mathrm{mm}$ for moderate and deep pockets, respectively.

In this manner, studies showing benefits of the photodynamic therapy usually presented poor results of the control treatment when compared to the ones reported by the present study. Andersen, et al. ${ }^{4}$ (2007), who obtained a statistical difference favoring PDT, observed a CAL gain of $0.86 \mathrm{~mm}$ and a reduction in $\mathrm{PD}$ of $1.11 \mathrm{~mm}$ for the group treated with SRP+PDT. The SRP group obtained a CAL gain of $0.36 \mathrm{~mm}$ and reduction in PD of $0.74 \mathrm{~mm}$, three months after treatment. Similarly, Braun, et al. ${ }^{7}$ (2008) observed a statistically significant reduction of the clinical parameters $\mathrm{PD}, \mathrm{BOP}, \mathrm{CAL}$ and salivary flow in the two groups with a high impact in the test group. The control group gained less attachment $(0.35 \mathrm{~mm})$ when compared with the test group $(0.67 \mathrm{~mm})$ and showed a smaller reduction in PD $(0.55 \mathrm{~mm}$ and $0.68 \mathrm{~mm}$ in the control and test group, respectively, considering the moderate pockets; $1.22 \mathrm{~mm}$ and $1.43 \mathrm{~mm}$ in the control and test group, respectively, considering the deep pockets). Although Braun, et al. ${ }^{7}$ (2008) found better results in the test group, the findings cannot be considered clinically significant. With regard to gingival recessions, there were no differences between the groups as found in the present study.

However, it is important to notice that comparison between studies are impaired by the differences in methods regarding periodontal diagnosis, study design, type and concentration of photosensitizer, laser type, configuration and protocol of application. When studying the effect of repeated applications of PDT with SRP in residual pockets of patients during supportive periodontal therapy (SPT), Lulic, et al. ${ }^{18}$ (2009) observed that 5 sessions of PDT associated with SRP significantly improved the PD, CAL and BOP after 6 months in the test group. However, even though Lulic, et al. ${ }^{18}$ (2009) suggests that successive applications of PDT with SRP may be recommended for treating residual pockets of patients in periodontal maintenance, the test group gained $0.52 \mathrm{~mm}$ in CAL and the control group lost $0.27 \mathrm{~mm}$ after 6 months. This discrepancy may have contributed for the appearance of a statistical difference between the groups.

Laser type is an important topic of discussion. PDT is characterized by the use of a photosensitizer in association with a low power laser (diode: GaAlAs, InGaAsP). This type of laser does not raise the intrapulp or periodontal ligament temperature, unlike high power lasers ${ }^{31}$. Therefore Zanin and Brugnera Jr ${ }^{31}$ (2007) stated that lowpower lasers are capable to restore cell biological balance, improving the conditions of tissue vitality.
They are also recognized by its analgesic, antiinflammatory and biomodulation effect on hard and soft tissues. Besides the physiological benefits, such lasers, when associated to a photosensitizer, have antimicrobial effects. The action mechanism that explains this feature is photochemical and photoelectric, resulting from the interaction of the dye with light. There is no thermal action, differently from the high power lasers that generally requires higher dosages of energy and reach the surface irradiated with high temperatures ${ }^{31}$. In this manner, Aoki, et al. ${ }^{5}$ (2004) demonstrated that a root surface with major thermal damage could render the tissue incompatible for normal cell attachment and healing.

According to Killoy ${ }^{14}$ (2002), clinical significance is an important parameter to assess the result of treatment. In this analysis, the percentage of sites with improvements $>2 \mathrm{~mm}$ in CAL and percentage of sites still requiring therapy have great importance. In the present study, the percentage of sites showing CAL gain of $\geq 2 \mathrm{~mm}$ after 6 months was $68.88 \%$ in the test group and $68.12 \%$ in control group. These results are similar to those found by Zanatta, et al. ${ }^{30}$ (2006) and higher than those found by Wennstrom, et al. ${ }^{29}$ (2005) and Del Peloso Ribeiro, et al. ${ }^{10}$ (2008).

In the present study, the amount of sites that needed retreatment after 3 months was $7.83 \%$ for the test group and $8.95 \%$ for the control group. After 6 months, these values were $4.51 \%$ and $4.16 \%$ in test and control group, respectively. There was no statistical difference among them, which confirm the good results obtained with the two therapies. These results are also similar to those found by Zanatta, et al. ${ }^{30}$ (2006) and Del Peloso Ribeiro, et al. ${ }^{10}$ (2008), but lower than those found by Wennstrom, et al. ${ }^{29}$ (2005), where all sites with a PD of $\geq 5 \mathrm{~mm}(42 \%)$ in the debridement group were qualified as needing retreatment. Thus, this difference is probably explained by the fact that, in the present study, only sites with a $\mathrm{PD} \geq 5 \mathrm{~mm}$ and BOP were retreated. This decision was taken because BOP is an important outcome measurement, since the absence of BOP in recall patients has been associated with clinical stability over time ${ }^{17}$. Furthermore, BOP associated with PPD has shown the greatest diagnostic accuracy for future attachment loss ${ }^{27}$.

The results of the present study are valid for nonsmoking patients with severe chronic periodontitis and with no systemic compromise. As a result, further studies with other types of periodontal diseases such as aggressive periodontitis, patients with systemic diseases, smokers, and patients with furcation lesions are needed to verify the existence of any additional effect of PDT associated with fullmouth ultrasonic debridement. 


\section{CONCLUSION}

Both treatments resulted in significant clinical improvements in the treatment of severe chronic periodontits, however PDT did not provide any additional benefit to those obtained with full-mouth ultrasonic debridement used alone.

\section{CONFLICT OF INTEREST STATEMENT}

The authors report no conflicts of interest related to this study.

\section{REFERENCES}

1- Ainamo J, Bay I. Problems and proposals for recording gingivitis and plaque. Int Dent J. 1975;25:229-35.

2- Almeida JM, Theodoro LH, Bosco AF, Nagata MJH, Oshiiwa $M$, Garcia VG. Influence of photodynamic therapy on the development of ligature-induced periodontitis in rats. J Periodontol. 2007;78:566-75.

3- Almeida JM, Theodoro LH, Bosco AF, Nagata MJH, Oshiiwa M, Garcia VG. In vivo effect of photodynamic therapy on periodontal bone loss in dental furcations. J Periodontol. 2008;79:1081-8.

4- Andersen R, Loebel N, Hammond D. Treatment of periodontal disease by photodisinfection compared to scaling and root planning. J Clin Dent. 2007;18:34-8.

5- Aoki A, Sasaki KM, Watanabe H, Ishikawa I. Laser in nonsurgical periodontal therapy. Periodontology 2000. 2004;36:59-97. 6- Atieh MA. Photodynamic therapy as an adjunctive treatment for chronic periodontitis: a meta-analysis. Lasers Med Sci. 2010;25: 605-13.

7- Braun A, Dehn C, Krause F, Jepsen S. Short-term effects of adjunctive antimicrobial photodynamic therapy in periodontal treatment: a randomized clinical trial. J Clin Periodontol. 2008;35:877-84.

8- Chondros P, Nikolidakis D, Christodoulides N, Rossler R, Gutknecht N, Sculean A. Photodynamic therapy as adjunct to non-surgical periodontal treatment in patients on periodontal maintenance: a randomized controlled clinical trial. Lasers Med Sci. 2009;24:668-88.

9- Christodoulides N, Nikolidakis D, Chondros P, Becker J, Schwarz $\mathrm{F}$, Rossler R, et al. Photodynamic therapy as an adjunct to nonsurgical periodontal treatment: a randomized, controlled clinical trial. J Periodontol. 2008;79:1638-44.

10- Del Peloso Ribeiro E, Bittencourt S, Sallum EA, Nociti FH Jr, Gonçalves RB, Casati MZ. Periodontal debridement as a therapeutic approach for severe chronic periodontitis: a clinical, microbiological and immunological study. J Clin Periodontol. 2008;35:789-98.

11- Flemmig TF. Periodontitis. Ann Periodontol. 1999;4:32-8.

12- Gonçalves PF, Gurgel BCV, Pimentel SP, Sallum EA, Sallum AW, Casati MZ, et al. Root cementum modulates periodontal regeneration in class III furcation defects treated by the guided tissue regeneration technique: a histometric study in dogs. J Periodontol. 2006;77:976-82.

13- Ishikawa I, Aoki A, Takasaki AA, Mizutani K, Sasaki KM, Izumi Y. Application of lasers in Periodontics: true innovation or myth? Periodontology 2000. 2009;50:90-126.
14- Killoy WJ. The clinical significance of local chemotherapies. J Clin Periodontol. 2002;29:22-9.

15- Komerik N, Wilson M, Poole S. The effect of photodynamic action on two virulence factors of gram-negative bacteria. Photochem Photobiol. 2000;72:676-80.

16- Komerik N, Nakanishi H, MacRobert AJ, Henderson B, Speight $\mathrm{P}$, Wilson $\mathrm{M}$. In vivo killing of Porphyromonas gingivalis by toluidine blue-mediated photosensitization in an animal model. Antimicrob Agents Chemother. 2003;47(3):932-40.

17- Lang NP, Adler R, Joss A, Nyman S. Absence of bleeding on probing. An indicator of periodontal stability. J Clin Periodontol. $1990 ; 17: 714-21$.

18- Lulic M, Gorog I, Salvi GE, Ramseier CA, Mattheos N, Lang NP. One-year of repeated adjunctive photodynamic therapy during periodontal maintenance: a proof-of-principle randomizedcontrolled clinical trial. J Clin Periodontol. 2009;36 661-6.

19- Muhlemann HR, Son S. Gingival sulcus bleeding a leading symptom in initial gingivitis. Helv Odontol Acta. 1971;15:107-13. 20- Nyman S, Wesfelt E, Sahed G, Karring T. Role of "diseased" root cementum in healing following treatment of periodontal disease. A clinical study. J Clin Periodontol. 1988;15:464-8.

21- Perussi JR. Inativação fotodinâmica de microrganismos. Quím Nova. 2007;30(4):988-94.

22- Pfitzner A, Sigush BW, Albrecht V, Glockmann E. Killing of periodontopathogenic bacteria by photodynamic therapy. J Periodontol. 2004;75:1343-9.

23- Polansky R, Haas M, Helschl A, Wimmer G. Clinical effectiveness of photodynamic therapy in the treatment of periodontitis. J Clin Periodontol. 2009;36:575-80.

24- Smart GJ, Wilson M, Davies EH, Kieser JB. Assessment of ultrasonic root surface debridement of residual endotoxin levels. J Clin Periodontol. 1990;1:174-8.

25- Sigush BW, Pfitzner A, Albrecht V, Glockmann E. Efficacy of photodynamic therapy on inflammatory signs and two selected periodontopathogenic species in a beagle dog model. J Periodontol. 2005;76:1100-5.

26- Takasaki AA, Aoki A, Mizutani K, Schwarz F, Sculean A, Wang $C Y$, et al. Application antimicrobial photodynamic therapy in periodontal and peri-implant diseases. Periodontology 2000. 2009;51:109-140.

27- Vanooteghem R, Hutchens LH, Garret S, Kiger R, Egelberg J. Bleeding on probing and probing depth as indicators of the response to plaque control and root debridement. J Clin Periodontol. 1987; 14:226-30.

28- Yilmaz S, Kuru B, Kuru L, Noyan U, Argun D, Kadir T. Effect of galium arsenide diode laser on human periodontal disease: a microbiological and clinical study. Lasers Surg Med. 2002;30:60-6. 29- Wennstrom JL, Tomasi C, Bertelle A, Dellasega E. Full-mouth ultra-sonic debridement versus quadrant scaling and root planing as an initial approach in the treatment of chronic periodontitis. J Clin Periodontol. 2005;32:851-9.

30- Zanatta GM, Bittencourt S, Nociti FH Jr, Sallum EA, Sallum AW, Casati MZ. Periodontal debridement with povidone-iodine in periodontal treatment short-term clinical and biochemical observations. J Periodontol. 2006;77:498-505.

31- Zanin ICJ, Brugnera A Jr. Terapia fotodinâmica no tratamento da doença periodontal. Perionews. 2007;1:79-85. 